\title{
Splitting schemes with respect to physical processes for double-porosity poroelasticity problems ${ }^{\text {th }}$
}

\author{
A.E. Kolesov ${ }^{\mathrm{a}}$, P.N. Vabishchevich ${ }^{\mathrm{b}, *}$ \\ ${ }^{a}$ North-Eastern Federal University, 58, Belinskogo, 677000 Yakutsk, Russia \\ ${ }^{b}$ Nuclear Safety Institute, Russian Academy of Sciences, 52, B. Tulskaya, 115191 Moscow, Russia
}

\begin{abstract}
We consider unsteady poroelasticity problem in fractured porous medium within the classical Barenblatt double-porosity model. For numerical solution of doubleporosity poroelasticity problems we construct splitting schemes with respect to physical processes, where transition to a new time level is associated with solving separate problem for the displacements and fluid pressures in pores and fractures. The stability of schemes is achieved by switching to three-level explicit-implicit difference scheme with some of the terms in the system of equations taken from the lower time level and by choosing a weight parameter used as a regularization parameter. The computational algorithm is based on the finite element approximation in space. The investigation of stability of splitting schemes is based on the general stability (well-posedness) theory of operator-difference schemes. A priori estimates for proposed splitting schemes and the standard two-level scheme are provided. The accuracy and stability of considered schemes are demonstrated by numerical experiments.
\end{abstract}

Keywords: poroelasticity, double-porosity, operator-difference schemes, splitting scheme, regularization

\section{Introduction}

Some aquifer and reservoir systems are formed by fractured rocks, which contain both pores and interconnected fractures. The fluid flow in such porous medium is most frequently modeled by the Barenblatt double-porosity model [4, 6], where we consider two diffusion processes coupled by an exchange term. Also, the pressures of fluid in both pores and fractures contribute to deformation of porous medium. Therefore, we incorporate Biot poroelasticity model [8] into Barenblatt model.

The mathematical model of double-porosity poroelasticity problem includes the Lame equation for the displacement and two nonstationary parabolic equations for the

\footnotetext{
This work was supported by the Russian Foundation for Basic Research (projects 14-01-00785).

${ }^{*}$ Correspondibg author.

Email addresses: kolesov.svfu@gmail .com (A.E. Kolesov), vabishchevich@gmail .com (P.N. Vabishchevich)
} 
fluid pressures in pores and fractures [7, 40]. Note that the displacement equation contains body forces, which are proportional to the gradient of pressures. In turn, the pressure equations includes dilation terms described by the divergence of the displacement velocity.

The numerical solution of poroelasticity problems is usually based on a finite element approximation in space [33, 38]. It is well-known that in order to satisfy LBBcondition (inf-sup condition) [13] we must use mixed finite elements, where the order of approximation for pressure is lower then the order of approximation for displacement. The finite element formulation of poroelasticity problem with double-porosity is presented in [23].

For the time approximation the standard two-level finite difference schemes with weights are used. In [11] the stability and convergence of two-level schemes for double-porosity poroelasticity problems with finite-difference approximations in space were analyzed using the general stability (correctness) theory of operator-difference schemes [34, 35]. The numerical implementation of two-level schemes is associated with solving coupled system of equations, which requires the use of special algorithms [1, 19] or additive operator-difference schemes (splitting schemes) [30, 36].

Splitting schemes are designed for the efficient computational realization of various unsteady problems by switching to a chain of simpler problems. The simplest example of such schemes is splitting with respect to spatial variables (alternating direction methods). Regionally additive schemes or domain decomposition methods are widely used for computations on parallel computers. For poroelasticity problems we use splitting with respect to physical processes, when the transition to a new time level is performed by sequentially solving separate problems for displacement and pressure.

Number of splitting schemes for poroelasticity problems are constructed and used in [3, 14, 15, 22, 24, 25, 27, 31, 39]. The analysis of stability and performance of these schemes is usually conducted using methodical computations without a theoretical study. Rigorous mathematical results concerning the stability of splitting schemes for poroelasticity problems are described in [37].

In this work, using Samarskii's regularization principle for operator-difference schemes we construct splitting schemes with respect to physical processes for doubleporosity poroelasticity problems. The paper is organized as follows. After a brief description of the mathematical problem in Section 2, we introduce the variational formulation of problem in Section 3. Here, we obtain a priori estimate that ensures the stability of the solution of problem. Discretization in space and time are performed in Sections 4 and 5, respectively. Splitting schemes are constructed in Sections 6 and 7. Numerical experiments that test the convergence and stability of considered schemes are shown in Section 8. Conclusions follow.

\section{Mathematical Model}

We consider double-porosity poroelasticity problems arising when a fluid flows through homogeneous and isotropic fractured medium where fractures and porous matrix represent two overlapping continua [4, 6]. In these continua two fluid flow processes with two different pressure fields occur. Therefore, the poroelasticity equations 
can be expressed as

$$
\begin{gathered}
\operatorname{div} \boldsymbol{\sigma}(\boldsymbol{u})-\alpha_{1} \operatorname{grad} p_{1}-\alpha_{2} \operatorname{grad} p_{2}=0, \\
\beta_{1} \frac{\partial p}{\partial t}+\alpha_{1} \frac{\partial \operatorname{div} \boldsymbol{u}}{\partial t}-\operatorname{div}\left(\frac{\boldsymbol{k}_{1}}{\eta} \operatorname{grad} p_{1}\right)+\gamma\left(p_{1}-p_{2}\right)=f_{1}(\boldsymbol{x}, t), \\
\beta_{2} \frac{\partial p}{\partial t}+\alpha_{2} \frac{\partial \operatorname{div} \boldsymbol{u}}{\partial t}-\operatorname{div}\left(\frac{\boldsymbol{k}_{2}}{\eta} \operatorname{grad} p_{2}\right)+\gamma\left(p_{2}-p_{1}\right)=f_{2}(\boldsymbol{x}, t) .
\end{gathered}
$$

Here, $\boldsymbol{u}$ is the displacement vector, $p_{1}$ is the fluid pressure in pores, and $p_{2}$ is fluid pressure in fractures. The stress tensor $\sigma$ is defined by the expression

$$
\boldsymbol{\sigma}=2 \mu \boldsymbol{\varepsilon}(\boldsymbol{u})+\lambda \operatorname{div} \boldsymbol{u} \boldsymbol{I},
$$

where $\mu$ is the shear modulus, $\lambda$ are the Lame coefficient, $\boldsymbol{I}$ is the unit tensor, and $\boldsymbol{\varepsilon}$ is the strain tensor:

$$
\boldsymbol{\varepsilon}=\frac{1}{2}\left(\operatorname{grad} \boldsymbol{u}+\operatorname{grad} \boldsymbol{u}^{T}\right) .
$$

The other notation is as follows: $\alpha$ is Biot coefficients, $\beta=1 / M, M$ is the Biot modulus, $\boldsymbol{k}_{1}, \boldsymbol{k}_{2}$ are the permeability tensors, $\eta$ is the viscosity of the fluid, $\gamma$ is the exchange parameter, and $f_{1}, f_{2}$ are the functions describing given fluid sources (sinks). The subscripts 1 and 2 represent notation associated with pores and fractures, respectively.

The system (11)- (3) is considered in a bounded domain $\Omega$ with a boundary $\Gamma$, on which, for simplicity, we set the following homogeneous conditions for displacements

$$
\boldsymbol{u}=0, \quad \boldsymbol{x} \in \Gamma_{D}, \quad \boldsymbol{\sigma n}=0, \quad \boldsymbol{x} \in \Gamma_{N} .
$$

For pressures we set

$$
\begin{array}{ll}
p_{1}=0, & \boldsymbol{x} \in \Gamma_{N}, \quad-\frac{\boldsymbol{k}_{1}}{\eta} \frac{\partial p_{1}}{\partial n}=0, \quad x \in \Gamma_{D}, \\
p_{2}=0, \quad \boldsymbol{x} \in \Gamma_{N}, \quad-\frac{\boldsymbol{k}_{2}}{\eta} \frac{\partial p_{2}}{\partial n}=0, \quad \boldsymbol{x} \in \Gamma_{D} .
\end{array}
$$

Here, $\boldsymbol{n}$ is the unit normal to the boundary, $\Gamma=\Gamma_{D}+\Gamma_{N}$. In addition, we specify the initial conditions for pressures as

$$
p_{1}(\boldsymbol{x}, 0)=s_{1}(\boldsymbol{x}), \quad p_{2}(\boldsymbol{x}, 0)=s_{2}(\boldsymbol{x}), \quad \boldsymbol{x} \in \Omega .
$$

The initial-boundary problem (17)-(7) for coupled parabolic and elliptic equations is the basis for considering fluid flow in deformable fractured porous medium.

\section{Variational Formulation}

For the numerical solution, we use finite element approximation in space [12, 26], so we need obtain a variational formulation of problem (17-(7). For scalar quantities, let us introduce the Hilbert space $L_{2}(\Omega)$ with the scalar product and norm given as

$$
(u, v)=\int_{\Omega} u(\boldsymbol{x}) v(\boldsymbol{x}), \quad\|u\|=(u, u)^{1 / 2} .
$$


For vector quantities, we use $\boldsymbol{L}_{2}(\Omega)=\left[L_{2}(\Omega)\right]^{m}$, where $m=2,3$ is the dimension of domain $\Omega$. Let $H^{1}(\Omega)$ and $\boldsymbol{H}^{1}(\Omega)$ be the Sobolev spaces. Next, we define the subspaces of scalar and vector functions

$$
\begin{aligned}
& Q=\left\{q \in H^{1}(\Omega): q(\boldsymbol{x})=0, \boldsymbol{x} \in \Gamma_{D}\right\}, \\
& \boldsymbol{V}=\left\{\boldsymbol{v} \in \boldsymbol{H}^{1}(\Omega): \boldsymbol{v}(\boldsymbol{x})=0, \boldsymbol{x} \in \Gamma_{D}\right\} .
\end{aligned}
$$

After multiplying (1), (2), and (3) by test functions $\boldsymbol{v} \in \boldsymbol{V}$ and $q_{1}, q_{2} \in Q$, respectively, and integrating by parts to eliminate the second order derivatives, we come to the following variational problem: Find $\boldsymbol{u} \in \boldsymbol{V}, p_{1}, p_{2} \in Q$ such that

$$
\begin{gathered}
a(\boldsymbol{u}, \boldsymbol{v})+\alpha_{1} g\left(p_{1}, \boldsymbol{v}\right)+\alpha_{2} g\left(p_{2}, \boldsymbol{v}\right)=0, \quad \forall \boldsymbol{v} \in \boldsymbol{V}, \\
c_{1}\left(\frac{\partial p_{1}}{\partial t}, q_{1}\right)+\alpha_{1} d\left(\frac{\partial \boldsymbol{u}}{\partial t}, q_{1}\right)+b_{1}\left(p_{1}, q_{1}\right)+\gamma\left(p_{1}-p_{2}, q_{1}\right)=\left(f_{1}, q_{1}\right), \quad \forall q_{1} \in Q, \\
c_{2}\left(\frac{\partial p_{2}}{\partial t}, q_{2}\right)+\alpha_{2} d\left(\frac{\partial \boldsymbol{u}}{\partial t}, q_{2}\right)+b_{2}\left(p_{2}, q_{2}\right)+\gamma\left(p_{2}-p_{1}, q_{2}\right)=\left(f_{2}, q_{2}\right), \quad \forall q_{2} \in Q .
\end{gathered}
$$

Here, the bilinear forms are defined as

$$
\begin{gathered}
a(\boldsymbol{u}, \boldsymbol{v})=\int_{\Omega} \boldsymbol{\sigma}(\boldsymbol{u}) \boldsymbol{\varepsilon}(\boldsymbol{v}) d \boldsymbol{x} \\
g(p, \boldsymbol{v})=\int_{\Omega} \operatorname{grad} p \boldsymbol{v} d \boldsymbol{x}, \quad d(\boldsymbol{u}, q)=\int_{\Omega} \operatorname{div} \boldsymbol{u} q d \boldsymbol{x} \\
c_{1}(p, q)=\beta_{1} \int_{\Omega} p q d \boldsymbol{x}, \quad c_{2}(p, q)=\beta_{2} \int_{\Omega} p q d \boldsymbol{x} \\
b_{1}(p, q)=\int_{\Omega} \frac{\boldsymbol{k}_{1}}{\eta} \operatorname{grad} p \operatorname{grad} q d \boldsymbol{x} \\
b_{2}(p, q)=\int_{\Omega} \frac{\boldsymbol{k}_{2}}{\eta} \operatorname{grad} p \operatorname{grad} q d \boldsymbol{x} .
\end{gathered}
$$

The initial conditions (7) are set as:

$$
\left(p_{1}(0), q_{1}\right)=\left(s_{1}, q_{1}\right), \quad\left(p_{2}(0), q_{2}\right)=\left(s_{2}, q_{2}\right), \quad q_{1}, q_{2} \in Q
$$

The bilinear form $a(\cdot, \cdot)$ is symmetric and positive definite:

$$
a(\boldsymbol{u}, \boldsymbol{v})=a(\boldsymbol{v}, \boldsymbol{u}), a(\boldsymbol{u}, \boldsymbol{u}) \geq \delta_{a}\|\boldsymbol{u}\|^{2}, \delta_{a}>0, \boldsymbol{u}, \boldsymbol{v} \in \boldsymbol{V}
$$

Taking into account this, the form is associated with a Hilbert space $H_{a}$ with the following inner product and norm:

$$
(\boldsymbol{u}, \boldsymbol{v})_{a}=a(\boldsymbol{v}, \boldsymbol{u}), \quad\|\boldsymbol{u}\|_{a}=(a(\boldsymbol{u}, \boldsymbol{u}))^{1 / 2} .
$$

The norm of the adjoint of the space $H_{a}$ is denoted by $\|\boldsymbol{u}\|_{*, a}$. Note, that forms $b_{1}(\cdot, \cdot)$, $b_{2}(\cdot, \cdot), c_{1}(\cdot, \cdot)$, and $c_{2}(\cdot, \cdot)$ are also symmetric and positive definite. 
According to the Green's formula, we have

$$
\int_{\Omega} \operatorname{div} \boldsymbol{u} p d \boldsymbol{x}=-\int_{\Omega} \operatorname{grad} p \boldsymbol{u} d \boldsymbol{x}+\int_{\Gamma} \boldsymbol{u n} p d \boldsymbol{x} .
$$

Then, in view of the boundary conditions $4-6$, forms $g(\cdot, \cdot)$ and $d(\cdot, \cdot)$ are related as

$$
d(\boldsymbol{u}, p)=-g(p, \boldsymbol{u}), \quad p \in Q, \quad \boldsymbol{u} \in \boldsymbol{V} .
$$

Now, we derive the simplest a priori estimates for the solution of problem 8 - 111 . Setting $\boldsymbol{v}=\partial \boldsymbol{u} / \partial t$ in $(8), q_{1}=p_{1}$ in (9), and $q_{2}=p_{2}$ in (10), summing up these equations and taking into account that, for example,

$$
a\left(\boldsymbol{u}, \frac{\partial \boldsymbol{u}}{\partial t}\right)=\frac{1}{2} \frac{d}{d t} a(\boldsymbol{u}, \boldsymbol{u})
$$

we obtain

$$
\begin{aligned}
\frac{1}{2} \frac{d}{d t}(a(\boldsymbol{u}, \boldsymbol{u}) & \left.+c_{1}\left(p_{1}, p_{1}\right)+c_{2}\left(p_{2}, p_{2}\right)\right)+b_{1}\left(p_{1}, p_{1}\right)+b_{2}\left(p_{2}, p_{2}\right) \\
& +\gamma\left\|p_{1}-p_{2}\right\|^{2}=\left(f_{1}, p_{1}\right)+\left(f_{2}, q_{2}\right) .
\end{aligned}
$$

For the right-hand side we use the following inequalities

$$
\left(f_{l}, p_{l}\right) \leq\left\|p_{l}\right\|_{b_{l}}^{2}+\frac{1}{4}\left\|f_{l}\right\|_{*, b_{l}}^{2}, \quad l=1,2
$$

Thus, we have

$$
\frac{d}{d t}\left(\|\boldsymbol{u}\|_{a}^{2}+\left\|p_{1}\right\|_{c_{1}}^{2}+\left\|p_{2}\right\|_{c_{2}}^{2}\right) \leq \frac{1}{2}\left(\left\|f_{1}\right\|_{*, b_{1}}^{2}+\left\|f_{1}\right\|_{*, b_{1}}^{2}\right)
$$

Using the initial condition $(11)$, we compute the initial displacement $\boldsymbol{u}_{0}(\boldsymbol{x})$

$$
a\left(\boldsymbol{u}_{0}, v\right)+\alpha_{1} g\left(s_{1}, v\right)+\alpha_{2} g\left(s_{2}, v\right)=0, \quad v \in V .
$$

Integration with respect to time gives the estimate:

$$
\begin{aligned}
\mid \boldsymbol{u}\left\|_{a}^{2}+\right\| p_{1} \|_{c_{1}}^{2} & +\left\|p_{2}\right\|_{c_{2}}^{2} \leq\left\|\boldsymbol{u}_{0}\right\|_{a}^{2}+\left\|s_{1}\right\|_{c_{1}}^{2} \\
& +\left\|s_{2}\right\|_{c_{2}}^{2}+\frac{1}{2} \int_{0}^{t}\left(\left\|f_{1}(\xi)\right\|_{*, b_{1}}^{2}+\left\|f_{2}(\xi)\right\|_{*, b_{2}}^{2}\right) d \xi
\end{aligned}
$$

using the notation $f(\cdot, t)=f(t)$.

A priori estimate $(12)$ ensures the stability of the solution of problem (8) - (11) with respect to the initial data and the right-hand side. Similar estimates can be obtained for the solution of discrete problem. 


\section{Finite Element Approximation}

Now, we approximate our problem in space using finite element methods. First, we construct a computational mesh $\Omega_{h}=\left\{\omega_{1}, \omega_{2}, \ldots, \omega_{N}\right\}$ of domain $\Omega$. Here, $N$ is the number of cells $\omega, h=\max _{\omega \in \Omega_{h}} h_{\omega}$, where $h_{\omega}$ is the diameter of circle inscribed in a cell $\omega$.

Then, in the mesh we define spaces of conformal finite elements for scalar and vector functions $Q_{h} \subset Q$ and $\boldsymbol{V}_{h} \subset \boldsymbol{V}$ and restrict the variational problem (8)-(11) to these spaces: find $\boldsymbol{u}_{h} \in \boldsymbol{V}_{h}$ and $p_{1, h}, p_{2, h} \in Q_{h}$ such that

$$
\begin{gathered}
a\left(\boldsymbol{u}_{h}, \boldsymbol{v}\right)+\alpha_{1} g\left(p_{1, h}, \boldsymbol{v}\right)+\alpha_{2} g\left(p_{2, h}, \boldsymbol{v}\right)=0, \forall \boldsymbol{v} \in \boldsymbol{V}_{h}, \\
c_{1}\left(\frac{\partial p_{1, h}}{\partial t}, q_{1}\right)+\alpha_{1} d\left(\frac{\partial \boldsymbol{u}_{h}}{\partial t}, q_{1}\right)+b_{1}\left(p_{1, h}, q_{1}\right) \\
+\gamma\left(p_{1, h}-p_{2, h}, q_{1}\right)=\left(f_{1}, q_{1}\right), q_{1} \in Q_{h}, \\
c_{2}\left(\frac{\partial p_{2, h}}{\partial t}, q_{2}\right) \\
+\alpha_{2} d\left(\frac{\partial \boldsymbol{u}_{h}}{\partial t}, q_{2}\right)+b_{2}\left(p_{2, h}, q_{2}\right) \\
+\gamma\left(p_{2, h}-p_{1, h}, q_{2}\right)=\left(f_{2}, q_{2}\right), q_{2} \in Q_{h}, \\
\left(p_{1, h}(0), q_{1}\right)=\left(s_{1}, q_{1}\right), \quad\left(p_{2, h}(0), q_{2}\right)=\left(s_{2}, q_{2}\right), q_{1}, q_{2} \in Q_{h} .
\end{gathered}
$$

For further consideration, it is convenient to use the operator formulation of problem (13) - 16). We define finite-dimensional operators $A, B_{1}, B_{2}, C_{1}, C_{2}, D, G$ related to the corresponding bilinear forms by setting, for example,

$$
\left(A \boldsymbol{u}_{h}, v\right)=a\left(\boldsymbol{u}_{h}, v\right), \quad \forall \boldsymbol{u}_{h}, v \in V_{h} .
$$

As a result, we come from problem $(13)-(16)$ to the following Cauchy problem for a system of equations:

$$
\begin{gathered}
A \boldsymbol{u}_{h}+\alpha_{1} G p_{1, h}+\alpha_{2} G p_{2, h}=0, \\
C_{1} \frac{d p_{1, h}}{d t}+\alpha_{1} D \frac{d \boldsymbol{u}_{h}}{d t}+B_{1} p_{1, h}+\gamma E\left(p_{1, h}-p_{2, h}\right)=f_{1, h}, \\
C_{2} \frac{d p_{2, h}}{d t}+\alpha_{2} D \frac{d \boldsymbol{u}_{h}}{d t}+B_{2} p_{2, h}+\gamma E\left(p_{2, h}-p_{1, h}\right)=f_{2, h}, \\
p_{1, h}(0)=s_{1, h}, \quad p_{2, h}(0)=s_{2, h} .
\end{gathered}
$$

where $E$ is the identity operator and

$$
\left(f_{l, h}, q_{l}\right)=\left(f_{l}, q_{l}\right),\left(s_{l, h}, q_{1}\right)=\left(s_{l}, q\right), \forall q_{l} \in Q_{h}, l=1,2 .
$$

The operator $A, B_{1}, B_{2}, C_{1}$ and $C_{2}$ are self-adjoint and positive definite, for example,

$$
A=A^{*}>0,
$$

while $D$ and $G$ are adjoint with to each other with an opposite sign:

$$
D=-G^{*}
$$


Note that for the operator formulation (17)- 20 , we can derive a discrete analogue of estimate (12):

$$
\begin{gathered}
\left\|\boldsymbol{u}_{h}\right\|_{A}^{2}+\left\|p_{1, h}\right\|_{C_{1}}^{2}+\left\|p_{2, h}\right\|_{C_{2}}^{2} \leq\left\|\boldsymbol{u}_{0, h}\right\|_{A}^{2}+\left\|s_{1, h}\right\|_{C_{1}}^{2}+\left\|s_{2, h}\right\|_{C_{2}}^{2} \\
+\frac{1}{2} \int_{0}^{t}\left(\left\|f_{1, h}(\xi)\right\|_{*, B_{1}}^{2}+\left\|f_{2, h}(\xi)\right\|_{*, B_{2}}^{2}\right) d \xi .
\end{gathered}
$$

Now, introducing a vector of pressures: $\boldsymbol{p}_{h}=\left\{p_{1, h}, p_{2, h}\right\}$, from 177-20 we obtain

$$
\begin{gathered}
A \boldsymbol{u}_{h}+\boldsymbol{G} \boldsymbol{p}_{h}=0, \\
\boldsymbol{C} \frac{d \boldsymbol{p}_{h}}{d t}+\boldsymbol{D} \frac{d \boldsymbol{u}_{h}}{d t}+\boldsymbol{B} \boldsymbol{p}_{h}=\boldsymbol{f}_{h}, \\
\boldsymbol{p}_{h}(0)=\boldsymbol{s}_{h} .
\end{gathered}
$$

Here

$$
\begin{aligned}
& \boldsymbol{G}=\left(\begin{array}{ll}
\alpha_{1} G & \alpha_{2} G
\end{array}\right), \quad \boldsymbol{D}=\left(\begin{array}{c}
\alpha_{1} D \\
\alpha_{2} D
\end{array}\right), \\
& \boldsymbol{C}=\left(\begin{array}{cc}
C_{1} & 0 \\
0 & C_{2}
\end{array}\right), \quad \boldsymbol{B}=\left(\begin{array}{cc}
B_{1}+\gamma E & -\gamma E \\
-\gamma E & B_{2}+\gamma E
\end{array}\right),
\end{aligned}
$$

and

$$
\boldsymbol{f}_{h}=\left\{f_{1, h}, f_{2, h}\right\}, \quad \boldsymbol{s}_{h}=\left\{s_{1, h}, s_{2, h}\right\} .
$$

The operators $\boldsymbol{C}$ and $\boldsymbol{B}$ are self-adjoint and positive definite, and $\boldsymbol{G}^{*}=-\boldsymbol{D}$ in the sense of the equality $\left(\boldsymbol{G} \boldsymbol{p}_{h}, \boldsymbol{u}_{h}\right)=-\left(\boldsymbol{p}_{h}, \boldsymbol{D} \boldsymbol{u}_{h}\right)$. Then, the solution of problem (22)-24) satisfies the a priori estimates

$$
\left\|\boldsymbol{u}_{h}\right\|_{A}^{2}+\left\|\boldsymbol{p}_{h}\right\|_{\boldsymbol{C}}^{2} \leq\left\|\boldsymbol{u}_{0, h}\right\|_{A}^{2}+\left\|\boldsymbol{s}_{h}\right\|_{\boldsymbol{C}}^{2}+\frac{1}{2} \int_{0}^{t}\left\|\boldsymbol{f}_{h}(\xi)\right\|_{*, \boldsymbol{B}}^{2} d \xi .
$$

Note that this estimate is similar to the apriori estimates (12), 21].

\section{Time Discretisation}

For the discretization in time we use a uniform grid with a step $\tau>0$. Let $\boldsymbol{u}^{n}=$ $\boldsymbol{u}_{h}\left(\boldsymbol{x}, t^{n}\right), \boldsymbol{p}^{n}=p_{h}\left(\boldsymbol{x}, t^{n}\right)$, where $t^{n}=n \tau, n=0,1, \ldots$ To obtain an approximate solution of problem (17)- $(20)$ we use the standard two-level scheme with weights:

$$
\begin{gathered}
A \boldsymbol{u}^{n+1}+\boldsymbol{G} \boldsymbol{p}^{n+1}=0, \\
\boldsymbol{C} \frac{\boldsymbol{p}^{n+1}-p^{n}}{\tau}+\boldsymbol{D} \frac{\boldsymbol{u}^{n+1}-\boldsymbol{u}^{n}}{\tau}+\boldsymbol{B} \boldsymbol{p}_{\theta}^{n+1}=\boldsymbol{f}_{\theta}^{n+1},
\end{gathered}
$$

where

$$
\boldsymbol{p}_{\theta}^{n+1}=\theta \boldsymbol{p}^{n+1}+(1-\theta) \boldsymbol{p}^{n}, \quad \boldsymbol{f}_{\theta}^{n+1}=\boldsymbol{f}_{h}\left(t_{\theta}^{n+1}\right)
$$

and $0 \leq \theta \leq 1$. The initial condition is written as

$$
\left(\boldsymbol{p}^{0}, \boldsymbol{q}\right)=(\boldsymbol{s}, \boldsymbol{q}) .
$$


Theorem 1. For $\theta \geq 0.5$ the solution of problem (26)-(28) satisfies the a priori estimate

$$
\left\|\boldsymbol{u}^{n+1}\right\|_{A}^{2}+\left\|\boldsymbol{p}^{n+1}\right\|_{C}^{2} \leq\left\|\boldsymbol{u}^{n}\right\|_{A}^{2}+\left\|\boldsymbol{p}^{n}\right\|_{\boldsymbol{C}}^{2}+\frac{\tau}{2}\left\|\boldsymbol{f}_{\theta}^{n+1}\right\|_{\boldsymbol{B}^{-1}}^{2}
$$

Proof. In view if linearity, multiplying 26 by $\frac{\boldsymbol{u}^{n+1}-\boldsymbol{u}^{n}}{\tau}$ and 27 by $p_{\theta}^{n+1}$, we get

$$
\begin{gathered}
\left(A \boldsymbol{u}_{\theta}^{n+1}, \frac{\boldsymbol{u}^{n+1}-\boldsymbol{u}^{n}}{\tau}\right)+\left(\boldsymbol{G} \boldsymbol{p}_{\theta}^{n+1}, \frac{\boldsymbol{u}^{n+1}-\boldsymbol{u}^{n}}{\tau}\right)=0 \\
\left(\boldsymbol{C} \frac{\boldsymbol{p}^{n+1}-\boldsymbol{p}^{n}}{\tau}, \boldsymbol{p}_{\theta}^{n+1}\right)+\left(\boldsymbol{D} \frac{\boldsymbol{u}^{n+1}-\boldsymbol{u}^{n}}{\tau}, \boldsymbol{p}_{\theta}^{n+1}\right)+\left(\boldsymbol{B} \boldsymbol{p}_{\theta}^{n+1}, \boldsymbol{p}_{\theta}^{n+1}\right)=\left(\boldsymbol{f}_{\theta}^{n+1}, \boldsymbol{p}_{\theta}^{n+1}\right),
\end{gathered}
$$

where

$$
\boldsymbol{u}_{\theta}^{n+1}=\theta \boldsymbol{u}^{n+1}+(1-\theta) \boldsymbol{u}^{n} .
$$

Summing up these equations, we obtain

$$
\left(A \boldsymbol{u}_{\theta}^{n+1}, \frac{\boldsymbol{u}^{n+1}-\boldsymbol{u}^{n}}{\tau}\right)+\left(\boldsymbol{C} \frac{\boldsymbol{p}^{n+1}-\boldsymbol{p}^{n}}{\tau}, \boldsymbol{p}_{\theta}^{n+1}\right)+\left(\boldsymbol{B} \boldsymbol{p}_{\theta}^{n+1}, \boldsymbol{p}_{\theta}^{n+1}\right)=\left(\boldsymbol{f}_{\theta}^{n+1}, \boldsymbol{p}_{\theta}^{n+1}\right) .
$$

Taking into account inequality

$$
\left(\boldsymbol{f}_{\theta}^{n+1}, \boldsymbol{p}_{\theta}^{n+1}\right) \leq\left\|\boldsymbol{p}_{\theta}^{n+1}\right\|_{\boldsymbol{B}}^{2}+\frac{1}{4}\left\|\boldsymbol{f}_{\theta}^{n+1}\right\|_{\boldsymbol{B}^{-1}}^{2},
$$

gives

$$
\left(A \boldsymbol{u}_{\theta}^{n+1}, \frac{\boldsymbol{u}^{n+1}-\boldsymbol{u}^{n}}{\tau}\right)+\left(\boldsymbol{C} \frac{\boldsymbol{p}^{n+1}-\boldsymbol{p}^{n}}{\tau}, \boldsymbol{p}_{\theta}^{n+1}\right) \leq \frac{1}{4}\left\|\boldsymbol{f}_{\theta}^{n+1}\right\|_{\boldsymbol{B}^{-1}}^{2}
$$

Using the equality

$$
\boldsymbol{v}_{\theta}^{n+1}=\theta \boldsymbol{v}^{n+1}+(1-\theta) \boldsymbol{v}^{n}=\frac{\boldsymbol{v}^{n+1}+\boldsymbol{v}^{n}}{2}+\left(\theta-\frac{1}{2}\right)\left(\boldsymbol{v}^{n+1}-\boldsymbol{v}^{n}\right)
$$

and taking into account that any self-adjoint operator $A$ satisfies

$$
(A(\boldsymbol{u}+\boldsymbol{v}), \boldsymbol{u}-\boldsymbol{v})=(A \boldsymbol{u}, \boldsymbol{u})-(A v, v),
$$

we obtain

$$
\begin{aligned}
\frac{1}{2}\left(\left\|\boldsymbol{u}^{n+1}\right\|_{A}^{2}-\left\|\boldsymbol{u}^{n}\right\|_{A}^{2}\right) & +\frac{1}{2}\left(\left\|\boldsymbol{p}^{n+1}\right\|_{\boldsymbol{C}}^{2}-\left\|\boldsymbol{p}^{n}\right\|_{\boldsymbol{C}}^{2}\right) \\
& +\left(\theta-\frac{1}{2}\right)\left(\left\|\boldsymbol{u}^{n+1}-\boldsymbol{u}^{n}\right\|_{A}^{2}+\left\|\boldsymbol{p}^{n+1}-\boldsymbol{p}^{n}\right\|_{\boldsymbol{C}}^{2}\right) \leq \frac{\tau}{4}\left\|\boldsymbol{f}_{\theta}^{n+1}\right\|_{\boldsymbol{B}^{-1}}^{2} .
\end{aligned}
$$

For $\theta \geq 0.5$, this yields the required estimate 29]. 
Note that in the numerical implementation of weighted scheme $\sqrt{26}-28 \boldsymbol{u}^{n+1}$, $p_{1}^{n+1}$, and $p_{2}^{n+1}$ are determined at each time level by solving the coupled system:

$$
\begin{gathered}
A \boldsymbol{u}^{n+1}+\alpha_{1} G p_{1}^{n+1}+\alpha_{2} G p_{2}^{n+1}=0 \\
C_{1} p_{1}^{n+1}+\alpha_{1} D \boldsymbol{u}^{n+1}+\theta \tau\left(B_{1}+\gamma E\right) p_{1}^{n+1}-\theta \tau \gamma E p_{2}^{n+1}=\psi_{1}^{n+1}, \\
C_{2} p_{2}^{n+1}+\alpha_{2} D \boldsymbol{u}^{n+1}+\theta \tau\left(B_{2}+\gamma E\right) p_{2}^{n+1}-\theta \tau \gamma E p_{1}^{n+1}=\psi_{2}^{n+1},
\end{gathered}
$$

with the corresponding $\psi_{1}^{n+1}$ and $\psi_{2}^{n+1}$. Various special numerical algorithms can be used to solve this system [1, 19].

Another opportunity is to construct splitting schemes:

$$
\begin{gathered}
A \boldsymbol{u}^{n+1}=\varphi_{\boldsymbol{u}}^{n+1}, \\
C_{1} p_{1}^{n+1}+\theta \tau B_{1} p_{1}^{n+1}=\varphi_{p_{1}}^{n+1}, \\
C_{2} p_{2}^{n+1}+\theta \tau B_{2} p_{2}^{n+1}=\varphi_{p_{2}}^{n+1},
\end{gathered}
$$

where the transition to a new time level involves the solution of separate equations for displacements and pressures.

\section{Incomplete Splitting Scheme}

To construct splitting schemes, we express $\boldsymbol{u}=\boldsymbol{u}_{h}$ from (22) and substitute the result into 23, which leads to a single equation for $\boldsymbol{p}=\boldsymbol{p}_{h}$

$$
-\boldsymbol{D} A^{-1} \boldsymbol{G} \frac{d \boldsymbol{p}}{d t}+\boldsymbol{C} \frac{d \boldsymbol{p}}{d t}+\boldsymbol{B} \boldsymbol{p}=\boldsymbol{f}
$$

This equation can be written as

$$
\widetilde{B} \frac{d p}{d t}+\widetilde{A} p=f
$$

where $\widetilde{\boldsymbol{A}}=\boldsymbol{B}$, and the operator $\widetilde{\boldsymbol{B}}$ is equal to sum of two self-adjoint, positive definite operators:

$$
\widetilde{\boldsymbol{B}}=\widetilde{\boldsymbol{B}}_{0}+\widetilde{\boldsymbol{B}}_{1}, \quad \widetilde{\boldsymbol{B}}_{0}=\boldsymbol{C}, \quad \widetilde{\boldsymbol{B}}_{1}=-\boldsymbol{D} A^{-1} \boldsymbol{G} .
$$

The operators $\widetilde{\boldsymbol{A}}, \widetilde{\boldsymbol{B}}$ are self-adjoint and positive definite.

Under the following constraint

$$
\widetilde{\boldsymbol{B}}_{1} \leq \gamma_{B} \widetilde{\boldsymbol{B}}_{0}, \quad \gamma_{B}>0
$$

for the numerical solution of problem (24), 30, we can use a thee-level explicitimplicit scheme with weights [18]:

$$
\widetilde{\boldsymbol{B}}_{0}\left(\theta \frac{\boldsymbol{p}^{n+1}-\boldsymbol{p}^{n}}{\tau}+(1-\theta) \frac{\boldsymbol{p}^{n}-\boldsymbol{p}^{n-1}}{\tau}\right)+\widetilde{\boldsymbol{B}}_{1} \frac{\boldsymbol{p}^{n}-\boldsymbol{p}^{n-1}}{\tau}+\widetilde{\boldsymbol{A}} \boldsymbol{p}^{n+1}=\boldsymbol{f}^{n+1}
$$


To calculate the first step, we can apply the two-level scheme

$$
\boldsymbol{p}^{0}=\boldsymbol{p}_{0}, \quad \widetilde{\boldsymbol{B}} \frac{\boldsymbol{p}^{1}-\boldsymbol{p}^{0}}{\tau}+\widetilde{\boldsymbol{A}} \boldsymbol{p}^{1}=\boldsymbol{f}^{1} .
$$

The value of $\theta$ is determined by the stability conditions for difference scheme (33), (34).

Taking into account equalities

$$
\begin{aligned}
& \frac{\boldsymbol{p}^{n+1}-\boldsymbol{p}^{n}}{\tau}= \frac{\boldsymbol{p}^{n+1}-\boldsymbol{p}^{n-1}}{2 \tau}+\frac{\tau}{2} \frac{\boldsymbol{p}^{n+1}-2 \boldsymbol{p}^{n}+\boldsymbol{p}^{n-1}}{\tau^{2}}, \\
& \frac{\boldsymbol{p}^{n}-\boldsymbol{p}^{n-1}}{\tau}= \frac{\boldsymbol{p}^{n+1}-\boldsymbol{p}^{n-1}}{2 \tau}-\frac{\tau}{2} \frac{\boldsymbol{p}^{n+1}-2 \boldsymbol{p}^{n}+\boldsymbol{p}^{n-1}}{\tau^{2}}, \\
& \boldsymbol{p}^{n+1}=\tau \frac{\boldsymbol{p}^{n+1}-\boldsymbol{p}^{n}}{\tau}+\boldsymbol{p}^{n},
\end{aligned}
$$

we can write the scheme 33 in the canonical form of three-level operator-difference schemes

$$
\widetilde{\boldsymbol{C}} \frac{\boldsymbol{p}^{n+1}-\boldsymbol{p}^{n-1}}{2 \tau}+\widetilde{\boldsymbol{D}} \frac{\boldsymbol{p}^{n+1}-2 \boldsymbol{p}^{n}+\boldsymbol{p}^{n-1}}{\tau^{2}}+\widetilde{\boldsymbol{A}} \boldsymbol{p}^{n}=\boldsymbol{f}^{n}
$$

where $\widetilde{\boldsymbol{C}}, \widetilde{\boldsymbol{D}}$ are the self-adjoint and positive definite operators given by

$$
\widetilde{\boldsymbol{C}}=\widetilde{\boldsymbol{B}}+\tau \widetilde{\boldsymbol{A}}, \quad \widetilde{\boldsymbol{D}}=\frac{\tau}{2}\left((2 \theta-1) \widetilde{\boldsymbol{B}}_{0}-\widetilde{\boldsymbol{B}}_{1}\right)+\frac{\tau^{2}}{2} \widetilde{\boldsymbol{A}} .
$$

Our subsequent analysis is based on the following general statement from the stability (well-posedness) theory of three-level operator-difference schemes [34, 35].

Lemma 1. For

$$
\widetilde{\boldsymbol{D}}>\frac{\tau^{2}}{4} \widetilde{\boldsymbol{A}},
$$

scheme (35) is unconditionally stable and its solution satisfies the estimate

$$
\mathcal{E}^{n+1} \leq \mathcal{E}^{n}+\frac{\tau}{2}\left\|\boldsymbol{f}^{n+1}\right\|_{\widetilde{\boldsymbol{C}}^{-1}}^{2},
$$

where

$$
\mathcal{E}^{n}=\left\|\frac{\boldsymbol{p}^{n}+\boldsymbol{p}^{n-1}}{2}\right\|_{\widetilde{\boldsymbol{A}}}^{2}+\left\|\frac{\boldsymbol{p}^{n}-\boldsymbol{p}^{n-1}}{\tau}\right\|_{\widetilde{\boldsymbol{D}}-\frac{\tau^{2}}{4} \widetilde{\boldsymbol{A}}}^{2} .
$$

Then, stability condition (37) holds if

$$
(2 \theta-1) \widetilde{\boldsymbol{B}}_{0}-\widetilde{\boldsymbol{B}}_{1} \geq 0 .
$$

In view of inequalities 32, this is achieved if we choose

$$
2 \theta \geq 1+\delta
$$


where $\delta$ is determined from (32) as the maximum eigenvalue of spectral problem $\delta=$ $v_{\max }$ :

$$
\widetilde{\boldsymbol{B}}_{1} \boldsymbol{p}=\nu \widetilde{\boldsymbol{B}}_{0} \boldsymbol{p}
$$

Taking into account (31) yields

$$
-D A^{-1} \boldsymbol{G} \boldsymbol{p}=\nu \boldsymbol{C p} .
$$

We set $\boldsymbol{u}=-A^{-1} \boldsymbol{G} \boldsymbol{p}$ and come to the problem

$$
A \boldsymbol{u}+\boldsymbol{G p}=0, \quad \boldsymbol{D u}=\nu \boldsymbol{C p} .
$$

Excluding $\boldsymbol{p}$, we have

$$
\boldsymbol{G C} \boldsymbol{C}^{-1} \boldsymbol{D} \boldsymbol{u}+v A \boldsymbol{u}=0 .
$$

Taking into account [25, we get the Cosserat spectrum problem [16, 32]

$$
\left(\alpha_{1}^{2}+\alpha_{2}^{2}\right) G \boldsymbol{C}^{-1} D \boldsymbol{u}+v A \boldsymbol{u}=0 .
$$

In principle, the maximum eigenvalue in 39 depends on the physical parameters of the differential problem $\left(\alpha_{i}, \beta_{i}, i=1,2, \mu, \lambda\right)$ and the domain, but not on the time steps.

Combining (31) and (33), we obtain the following incomplete splitting scheme:

$$
\begin{gathered}
A \boldsymbol{u}^{n+1}+\boldsymbol{G} \boldsymbol{p}^{n}=0, \\
\boldsymbol{C}\left(\theta \frac{\boldsymbol{p}^{n+1}-\boldsymbol{p}^{n}}{\tau}+(1-\theta) \frac{\boldsymbol{p}^{n}-\boldsymbol{p}^{n-1}}{\tau}\right)+\boldsymbol{D} \frac{\boldsymbol{u}^{n+1}-\boldsymbol{u}^{n}}{\tau}+\boldsymbol{B} \boldsymbol{p}^{n+1}=\boldsymbol{f}^{n+1},
\end{gathered}
$$

where the solution is determined by solving separately the elasticity problem (40) and the double porosity problem 41 . The stability of this splitting scheme can be described by the following theorem:

Theorem 2. Scheme (40), (41) is unconditionally stable for $2 \theta \geq 1+\delta$, where $\delta=v_{\max }$ is the maximum eigenvalue of spectral problem (39).

\section{Full Splitting Scheme}

In the splitting scheme with respect to physical processes 40 , (41) on each time level we solve two separate problems: the displacement problem (40) and the coupled problem for pressures in pores and fractures (41). It makes sense to build a splitting scheme, where we have separate problems for pressures as well.

We can split the scheme 30 further separating the diagonal part of operator $\widetilde{\boldsymbol{A}}$ as follows

$$
\widetilde{A}=\widetilde{A}_{0}+\widetilde{A_{1}}
$$

where

$$
\widetilde{\boldsymbol{A}}_{0}=\left(\begin{array}{cc}
B_{1}+\gamma E & 0 \\
0 & B_{2}+\gamma E
\end{array}\right), \quad \widetilde{\boldsymbol{A}}_{1}=\left(\begin{array}{cc}
0 & -\gamma E \\
-\gamma E & 0
\end{array}\right) .
$$

Under the assumption

$$
\widetilde{A_{0}}-\widetilde{A_{1}} \geq 0
$$


which follows from positive-definiteness of the operator $\widetilde{A}$, we can use the following explicit-implicit scheme

$$
\begin{aligned}
& \widetilde{\boldsymbol{B}}_{0}\left(\theta \frac{\boldsymbol{p}^{n+1}-\boldsymbol{p}^{n}}{\tau}+(1-\theta) \frac{\boldsymbol{p}^{n}-\boldsymbol{p}^{n-1}}{\tau}\right) \\
& +\widetilde{\boldsymbol{B}}_{1} \frac{\boldsymbol{p}^{n}-\boldsymbol{p}^{n-1}}{\tau}+\widetilde{\boldsymbol{A}}_{0} \boldsymbol{p}^{n+1}+\widetilde{\boldsymbol{A}}_{1} \boldsymbol{p}^{n}=\boldsymbol{f}^{n+1} .
\end{aligned}
$$

Similar to (33) scheme (44) can be written in the canonical form of three-level operator difference schemes 35 with

$$
\widetilde{\boldsymbol{C}}=\widetilde{\boldsymbol{B}}+\tau \widetilde{\boldsymbol{A}_{0}}, \quad \widetilde{\boldsymbol{D}}=\frac{\tau}{2}\left((2 \theta-1) \widetilde{\boldsymbol{B}}_{0}-\widetilde{\boldsymbol{B}}_{1}\right)+\frac{\tau^{2}}{2} \widetilde{\boldsymbol{A}_{0}},
$$

and the analysis of the resulting scheme can be based on Lemma 1. In this case, the stability condition (37) takes the form

$$
2\left((2 \theta-1) \widetilde{\boldsymbol{B}}_{0}-\widetilde{\boldsymbol{B}}_{1}\right)+\tau\left(2 \widetilde{\boldsymbol{A}}_{0}-\widetilde{\boldsymbol{A}}\right)>0 .
$$

Taking into account (42), (43), the stability condition becomes $2 \theta \geq 1+\delta$, where $\delta$ is determined from 39 .

Finally, combining [31), (42), and (44), we have the full splitting scheme:

$$
\begin{gathered}
A \boldsymbol{u}^{n+1}+\alpha_{1} G p_{1}^{n}+\alpha_{2} G p_{2}^{n}=0, \\
\alpha_{1} D \frac{\boldsymbol{u}^{n+1}-\boldsymbol{u}^{n}}{\tau}+ \\
+B_{1} p_{1}^{n+1}+\gamma\left(p_{1}^{n+1}-p_{2}^{n}\right) \\
+C_{1}\left(\theta \frac{p_{1}^{n+1}-p_{1}^{n}}{\tau}+(1-\theta) \frac{p_{1}^{n}-p_{1}^{n-1}}{\tau}\right)=f_{1}^{n+1}, \\
\alpha_{2} D \frac{\boldsymbol{u}^{n+1}-\boldsymbol{u}^{n}}{\tau}+ \\
+B_{2} p_{2}^{n+1}+\gamma\left(p_{2}^{n+1}-p_{1}^{n}\right) \\
+C_{2}\left(\theta \frac{p_{2}^{n+1}-p_{2}^{n}}{\tau}+(1-\theta) \frac{p_{2}^{n}-p_{2}^{n-1}}{\tau}\right)=f_{2}^{n+1} .
\end{gathered}
$$

Here, the solution is determined by solving each equation separately. We can formulate a similar theorem, which describes the stability of this scheme:

Theorem 3. Splitting scheme (45)-(47) is unconditionally stable for $2 \theta \geq 1+\delta$, where $\delta=v_{\max }$ is the maximum eigenvalue of spectral problem 39.

\section{Numerical Experiment}

We consider a two-dimensional double-porosity poroelasticity problem in a unit square domain $\Omega$ subjected to a surface load $\boldsymbol{g}$ as in Fig. 1] At some centered upper part of the domain $\Gamma_{1}$ the load $\boldsymbol{g}=-\sin (\pi t) \boldsymbol{n}$ is applied in strip of length $0.2 \mathrm{~m}$. The remaining of the top boundary $\Gamma_{2}$ is traction free. On the vertical boundaries $\Gamma_{3}$ we set zero horizontal displacement and vertical surface traction. The bottom of the domain $\Gamma_{4}$ is assumed to be fixed, i.e. the displacement vector is taken as zero. For pressures, 
we prescribe both pore and fracture pressure to be zero at $\Gamma_{2}$, while the remaining boundaries are impermeable. More precisely, the boundary conditions are given as follows

$$
\begin{aligned}
& p_{1}=0, \quad p_{2}=0, \quad \boldsymbol{\sigma} \boldsymbol{n}=0, \quad \boldsymbol{x} \in \Gamma_{1}, \\
& \frac{\partial p_{1}}{\partial n}=0, \quad \frac{\partial p_{2}}{\partial n}=0, \quad \boldsymbol{\sigma n}=\boldsymbol{g}, \quad \boldsymbol{x} \in \Gamma_{2}, \\
& \frac{\partial p_{1}}{\partial n}=0, \quad \frac{\partial p_{2}}{\partial n}=0, \quad(\boldsymbol{\sigma n}) \times \boldsymbol{n}=0, \quad \boldsymbol{u n}=0, \boldsymbol{x} \in \Gamma_{3}, \\
& \frac{\partial p_{1}}{\partial n}=0, \quad \frac{\partial p_{2}}{\partial n}=0, \quad \boldsymbol{u}=0, \quad \boldsymbol{x} \in \Gamma_{4} .
\end{aligned}
$$

Similar mathematical model is commonly used to test computational algorithms for numerical solution of poroelasticity problems [9, 10, 17].

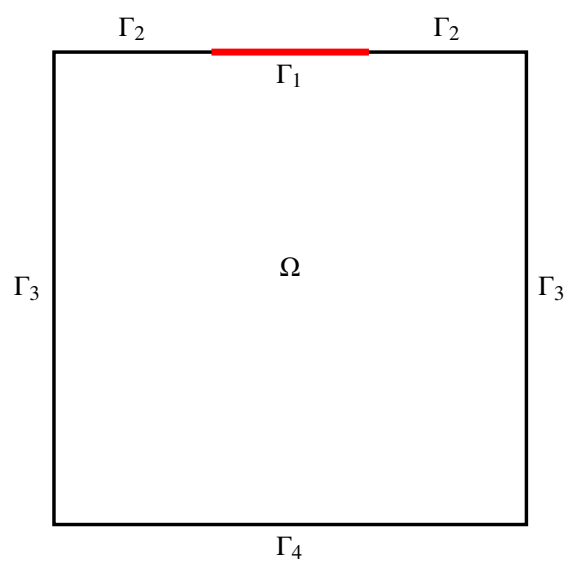

Figure 1: Computational domain

To analyze the considered numerical schemes and to identify the dependence of the stability of splitting schemes from the problem parameters we use three sets of input parameters presented in Table 1 . For convenience, we vary only values of the $\beta_{1}$ and $\beta_{2}$, and other parameters are the same for all sets.

For the numerical solution we use four computational meshes of different quality with the local refinement in the area of application of the traction. The numbers of vertices, cells, and degrees of freedom (dof) of $\boldsymbol{u}, p_{1}, p_{2}$, and $\boldsymbol{w}=\left\{\boldsymbol{u}, p_{1}, p_{2}\right\}$ for each mesh are given in Table 2 Fig 2 shows the coarse mesh 1 . Here, the quadratic vector element and the linear scalar element are used for discretization of the displacement and pressures, respectively. Therefore, the number of dofs for the the displacement $\boldsymbol{u}$ is much higher than the dofs number for the pressures $p_{1}$ and $p_{2}$.

The numerical implementation is performed using the DOLFIN library, which is a part of the FEniCS project for automated solution of differential equations by finite element methods [2, 28, 29]. The computational meshes are generated using the Gmsh 
Table 1: Problem properties

\begin{tabular}{ccccc}
\hline Parameter & Unit & Set 1 & Set 2 & Set 3 \\
\hline$\eta$ & $\mathrm{Pa} \cdot \mathrm{s}$ & 0.001 & 0.001 & 0.001 \\
$\mu$ & $\mathrm{MPa}$ & 4.2 & 4.2 & 4.2 \\
$\lambda$ & $\mathrm{MPa}$ & 2.4 & 2.4 & 2.4 \\
$\beta_{1}$ & $\mathrm{GPa}^{-1}$ & 54 & 108 & 216 \\
$\beta_{2}$ & $\mathrm{GPa}^{-1}$ & 14 & 24 & 48 \\
$k_{1}$ & $10^{-15} \mathrm{~m}^{2}$ & 6.18 & 6.18 & 6.18 \\
$k_{2}$ & $10^{-15} \mathrm{~m}^{2}$ & 27,2 & 27.2 & 27.2 \\
$\alpha_{1}$ & - & 0.95 & 0.95 & 0.95 \\
$\alpha_{2}$ & - & 0.12 & 0.12 & 0.12 \\
$\gamma$ & $10^{-10} \mathrm{~kg} /(\mathrm{m} \cdot \mathrm{s})$ & 5 & 5 & 5 \\
\hline
\end{tabular}

software [20]. To solve systems of linear equations we use LU solver provided by PETSc [5].

Table 2: Parameters of meshes

\begin{tabular}{lrrrr}
\hline & mesh 1 & mesh 2 & mesh 3 & mesh 4 \\
\hline Vertices & 881 & 3409 & 13398 & 52366 \\
Cells & 1654 & 6604 & 26370 & 103884 \\
Number of $\boldsymbol{u}$ dof & 6830 & 26842 & 106330 & 417230 \\
Number of $p_{1}$ dof & 881 & 3409 & 13398 & 52366 \\
Number of $p_{2}$ dof & 881 & 3409 & 13398 & 52366 \\
Number of $\boldsymbol{w}$ dof & 8592 & 33660 & 133126 & 521962 \\
\hline
\end{tabular}

Fig. 3- 5 show the displacement and pressures in pores and fractures, respectively, at time $t=0.5 \mathrm{~s}$, which are obtained using the two-level scheme $(26)-(28)$ with $\theta=1.0$ on the finest mesh 4 and time step $\tau=0.0025 \mathrm{~s}$. The displacement is presented on the deformed domain (overstated for visual contrast). We see that the pressure in fractures declines more rapidly than in pores. These results will be used as etalon solutions $\boldsymbol{u}_{e}$, $p_{1, e}$, and $p_{2, e}$ to evaluate errors as

$$
\varepsilon_{\boldsymbol{u}}=\left\|\boldsymbol{u}_{e}-\boldsymbol{u}\right\|, \quad \varepsilon_{p_{1}}=\left\|p_{1, e}-p_{1}\right\|, \quad \varepsilon_{p_{2}}=\left\|p_{2, e}-p_{2}\right\|
$$

We analyze the dependence of the accuracy of two-level scheme with weights (26)28) from computational parameters: mesh and time step sizes. On this stage, we employ only the first set of input parameters (Table. 1), because they do not have an impact on the computational character of two-level scheme.

Fig. 6 illustrates the dynamics of errors of the pressure in pores $\varepsilon_{p_{1}}$ for different meshes 1,2 , and 3 with $\tau=0.0025 \mathrm{~s}$, while errors for different time steps $\tau=0.02$, 0.01 , and $0.005 \mathrm{~s}$ on mesh 3 are shown in Fig. 7. The weight $\theta=1.0$ is used. We 


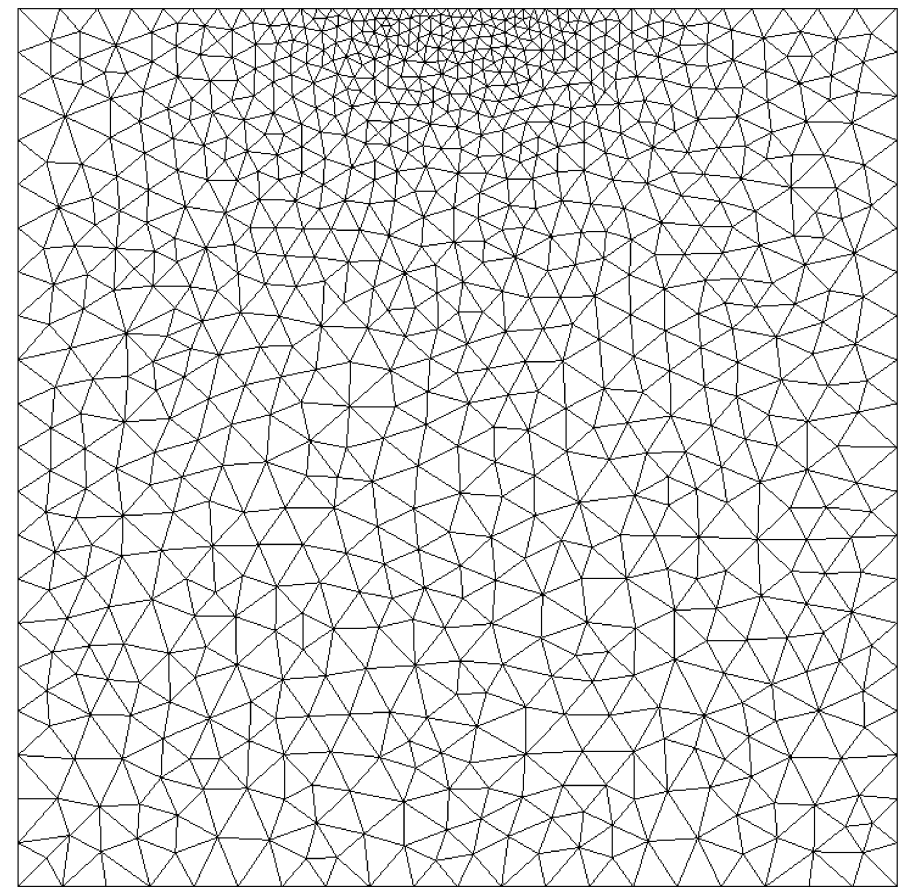

Figure 2: Computational mesh 1

observe the convergence of solution when increasing quality of mesh and reducing time step $\tau$.

Now, we conduct experiments to demonstrate the efficiency of incomplete splitting scheme (40)-[41). First, using the SLEPs library [21] we solve the eigenvalue problem (39) and find the value of $\delta=v_{\max }$ and estimate the minimum value of the weight $\theta$ using (38), when three-level scheme is unconditionally stable. The results are presented in Table 3. Note that the eigenvalues $v_{\max }$ are virtually independent from mesh size and depend only on the problem properties.

In Figs. 8-10 we present the dynamics of errors $\varepsilon_{p_{1}}$ for different values of $\theta$ for sets 1,2 , and 3, respectively. In all cases, if the condition (38) is not satisfied, then the

Table 3: Parameter $\delta$ and weight $\theta$

\begin{tabular}{llll}
\hline & Set 1 & Set 2 & Set 3 \\
\hline$\delta$ & 2.49 & 1.25 & 0.62 \\
$\theta$ & 1.75 & 1.12 & 0.81 \\
\hline
\end{tabular}




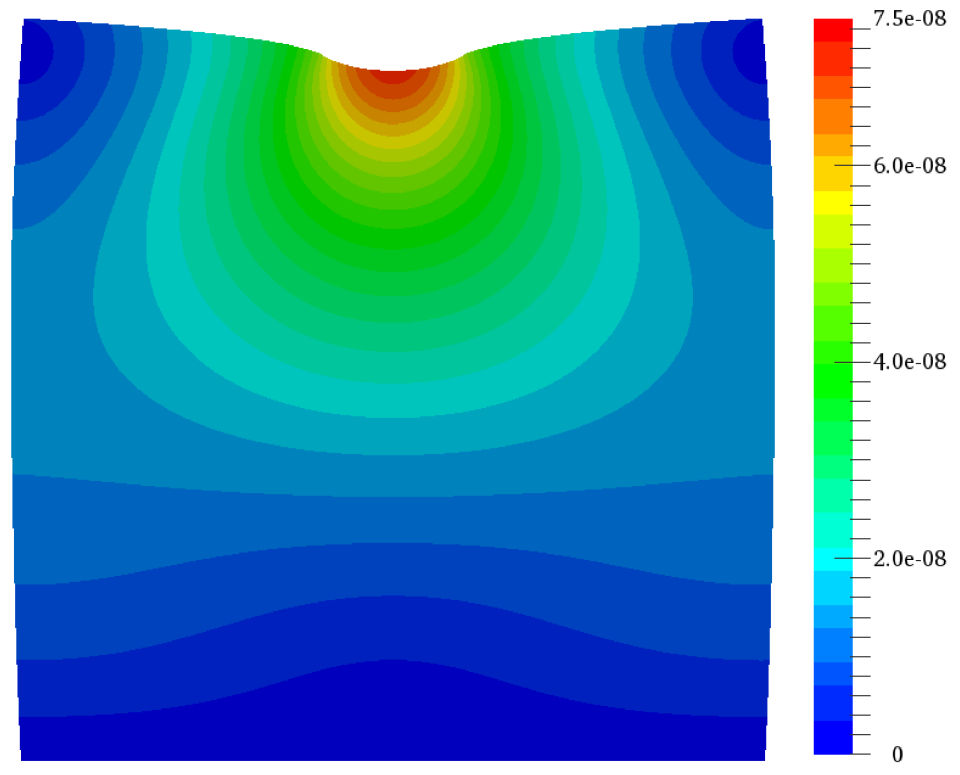

Figure 3: Distribution of displacement

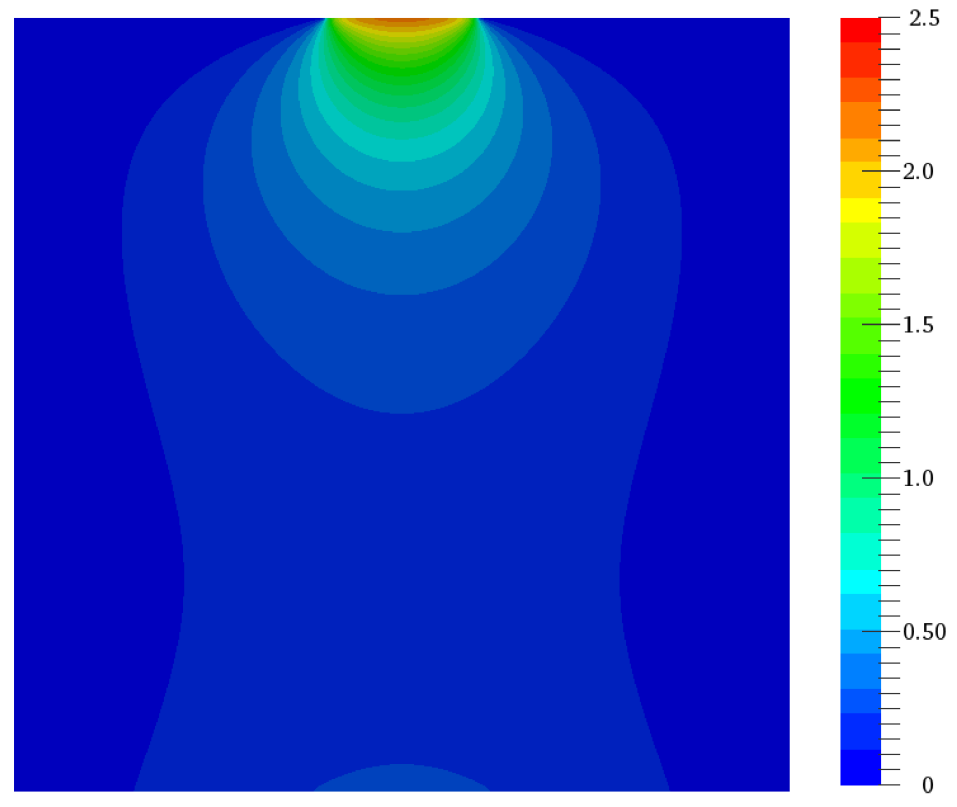

Figure 4: Distribution of pressures in pores 


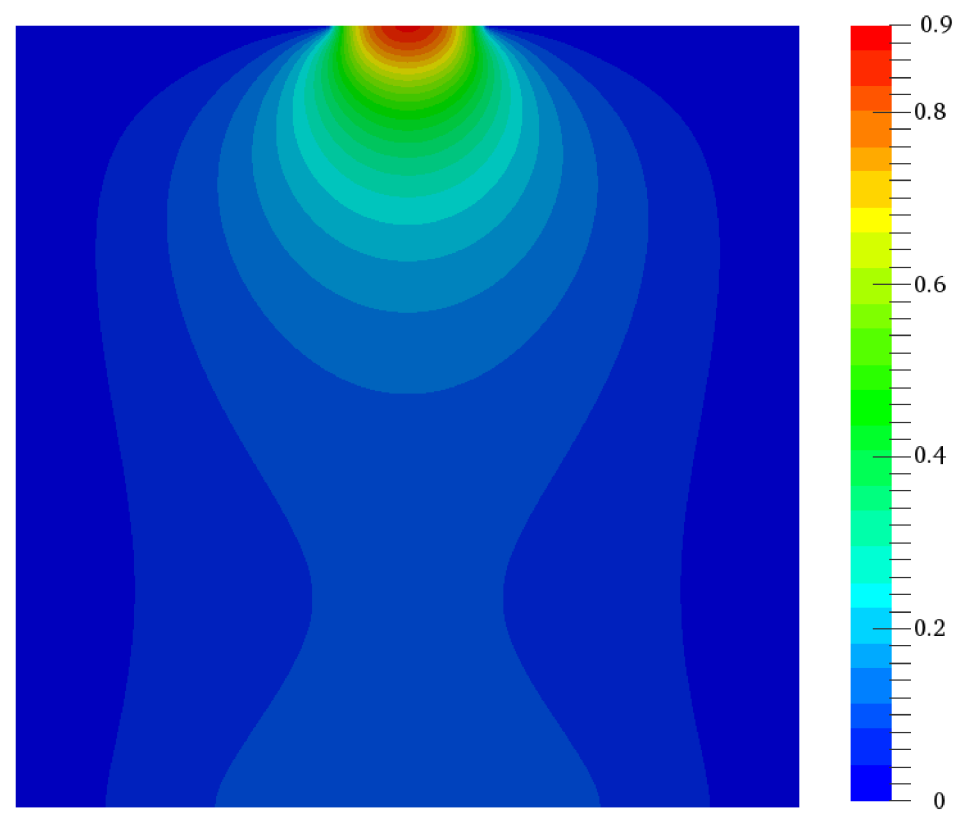

Figure 5: Distribution of pressures in fractures

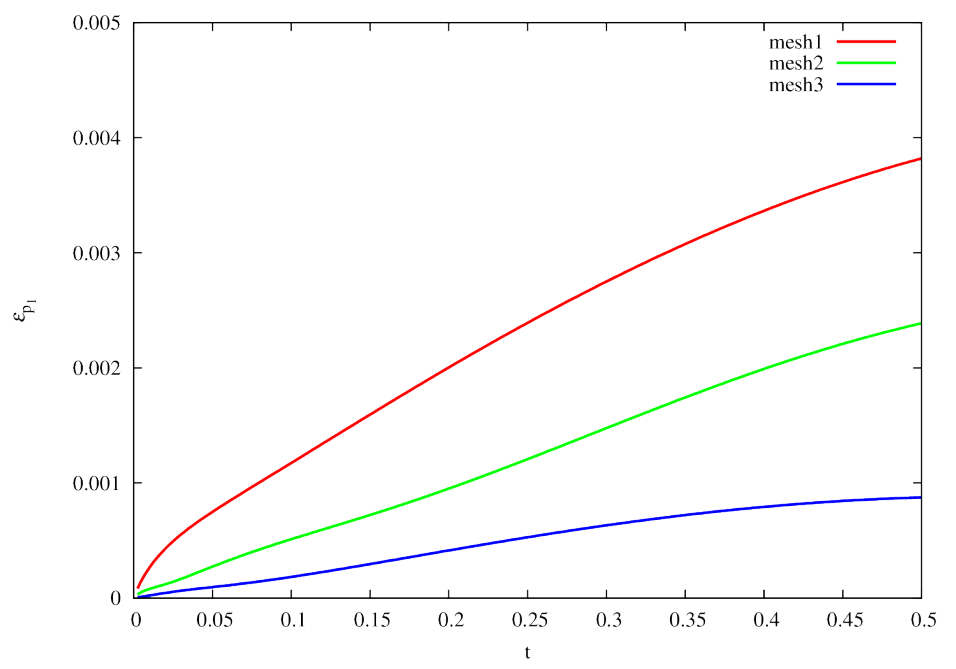

Figure 6: Coupled scheme: comparison of the errors $\varepsilon_{p_{1}}$ for different meshes

solution is unstable. When the value of the weight is taken according to Table 3 , the solution is regularized. 


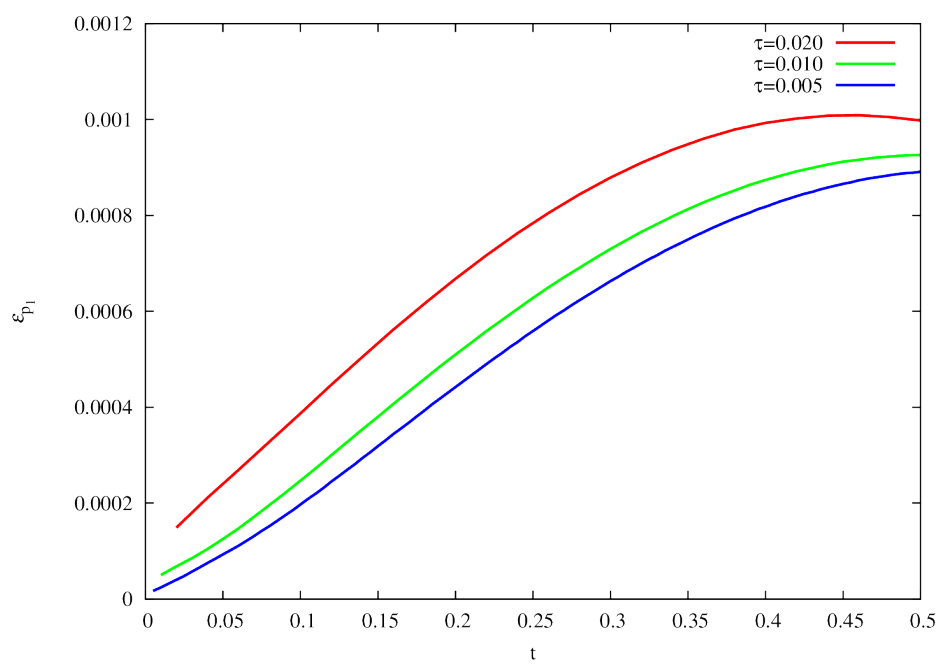

Figure 7: Coupled scheme: comparison of the errors $\varepsilon_{p_{1}}$ for different time steps

Table 4: Solve time for different meshes and numerical schemes

\begin{tabular}{lrrr}
\hline & $\begin{array}{r}\text { Full splitting } \\
\text { scheme }\end{array}$ & $\begin{array}{r}\text { Incomplete } \\
\text { splitting scheme }\end{array}$ & $\begin{array}{r}\text { Coupled } \\
\text { scheme }\end{array}$ \\
\hline mesh 1 & 6.894 & 7.045 & 12.684 \\
mesh 2 & 27.578 & 28.452 & 55.892 \\
mesh 3 & 154.824 & 165.958 & 305.932 \\
mesh 4 & 866.452 & 1033.207 & 2488.546 \\
\hline
\end{tabular}

Next, we consider the full splitting scheme (45)-477). The dynamics of errors of the pressure in fractures $\varepsilon_{p_{2}}$ for different meshes and time steps are shown in Figs. 11 and 12. Here, we use the first set of input parameters (Table 1) and $\theta=1.8$. We also see good convergence of solution.

Finally, in Table 4 we present the dependence of solve time of coupled scheme 26, (27), incomplete splitting scheme (40), (41), and full splitting scheme (45)-(47) from mesh size. Here, the first set of input parameters and $\tau=0.005$ are used. We see that splitting schemes are significantly faster than coupled scheme. When mesh is small, the solve times of the incomplete and full splitting schemes differ little from each other. When mesh is big, the full splitting scheme is much faster than incomplete scheme.

\section{Conclusion}

In this paper, we constructed unconditionally stable three-level splitting schemes with weights for numerical solution of double-porosity poroelasticity problems. The 


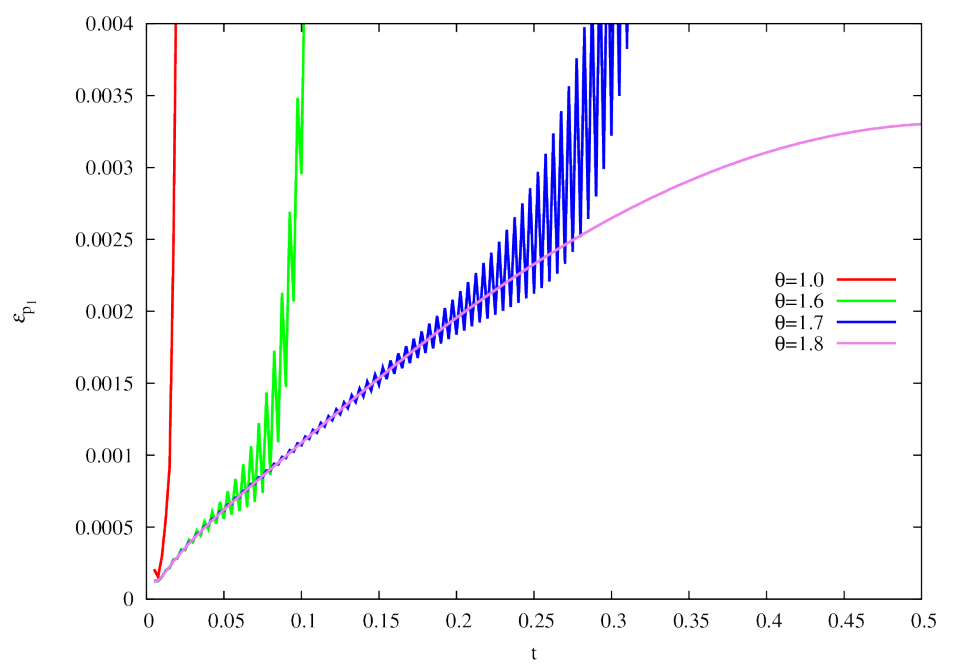

Figure 8: Incomplete splitting scheme: comparison of the errors $\varepsilon_{p_{1}}$ for different $\theta$ and Set 1

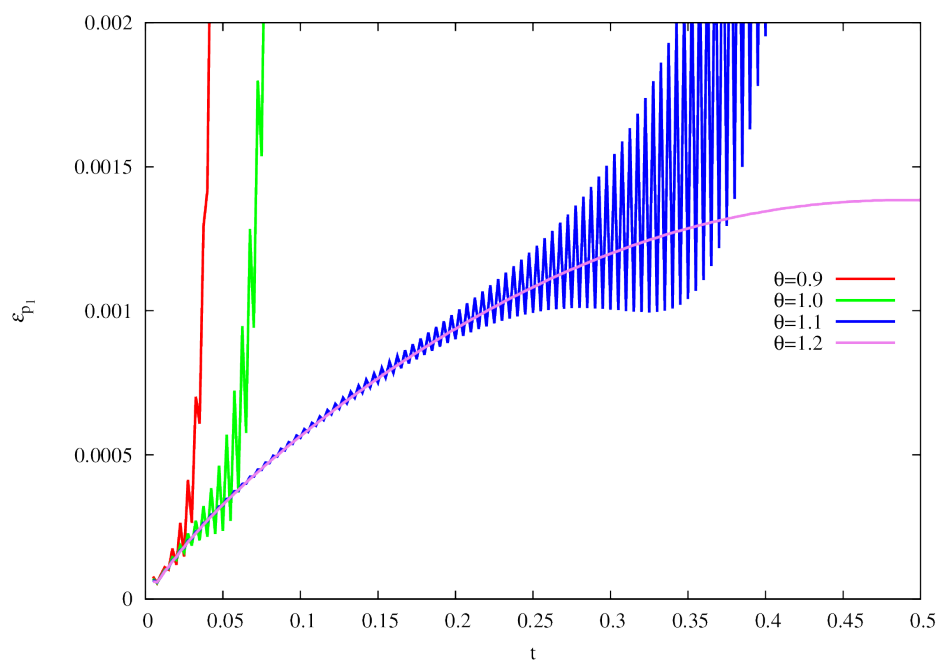

Figure 9: Incomplete Splitting scheme. Comparison of the errors $\varepsilon_{p_{1}}$ for different $\theta$ and Set 2 


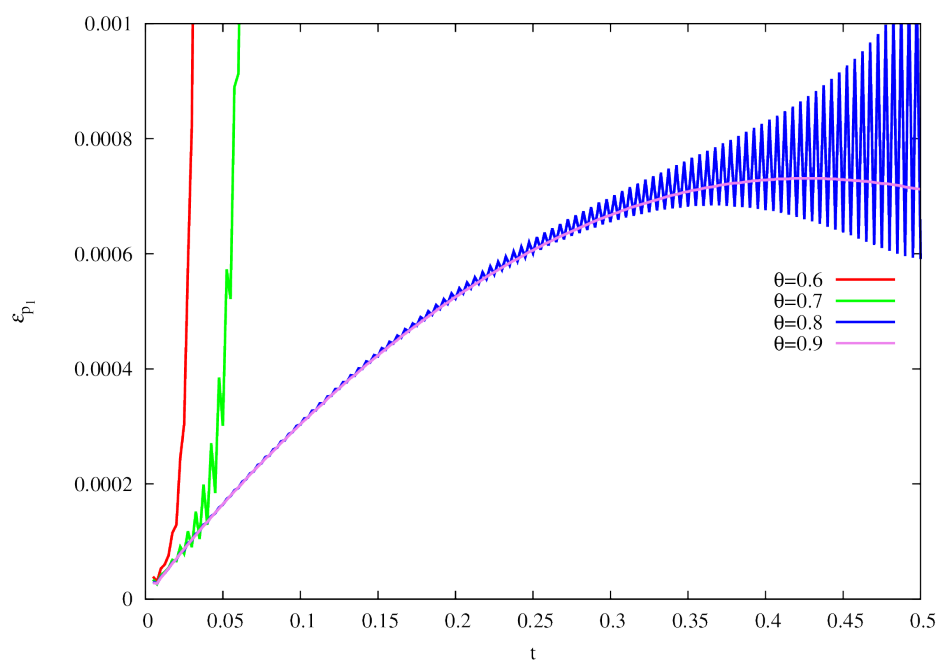

Figure 10: Incomplete splitting scheme: comparison of the errors $\varepsilon_{p_{1}}$ for different $\theta$ and Set 3

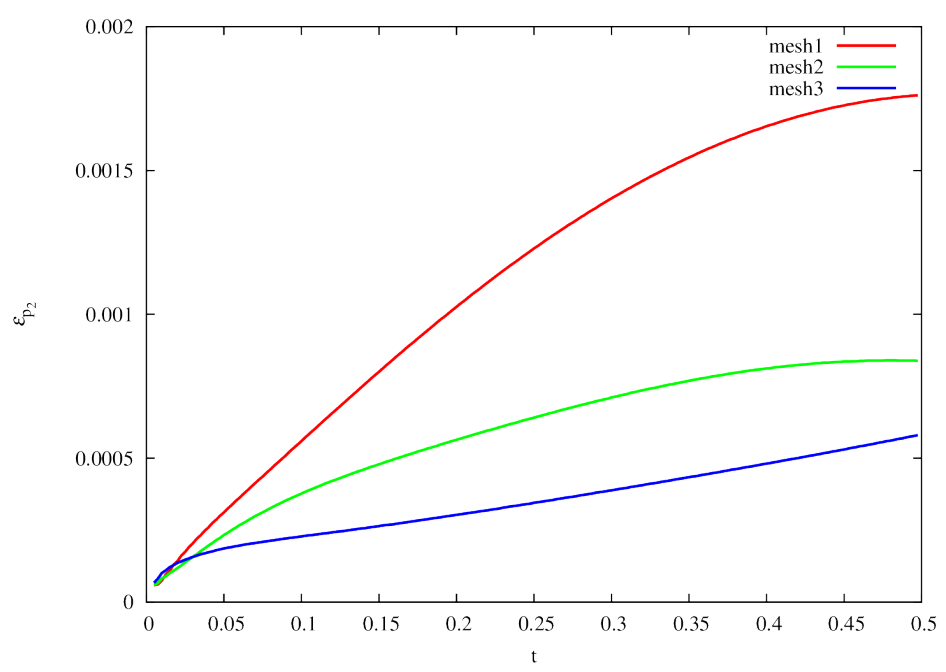

Figure 11: Full splitting scheme: comparison of the errors $\varepsilon_{p_{2}}$ for different meshes

analysis was based on the general theory of stability and correctness of operatordifference schemes. The finite element method was used approximation in space. The efficiency of considered schemes were verified by numerical experiments. 


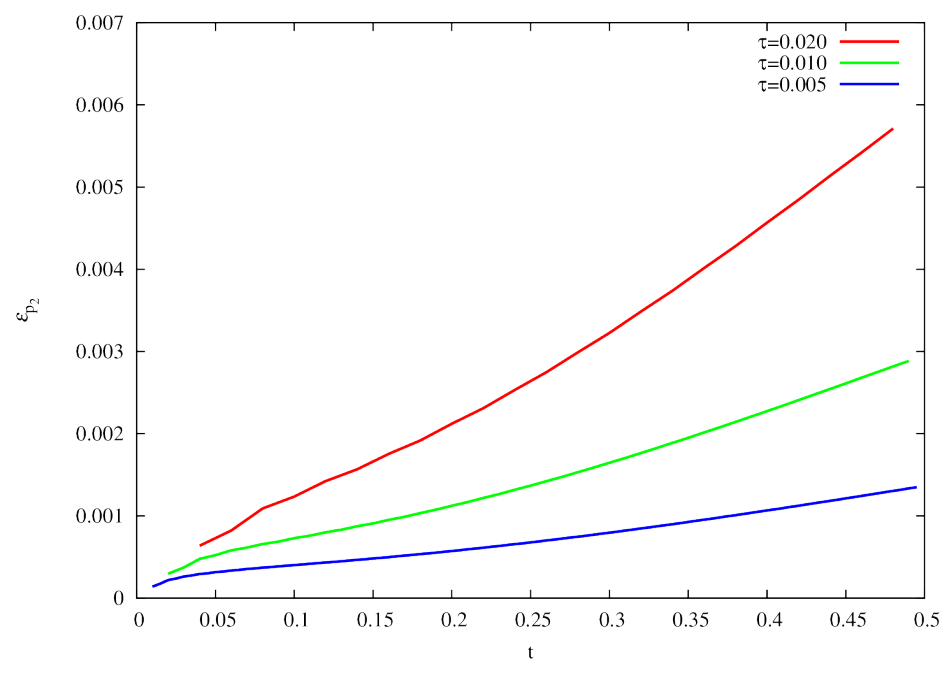

Figure 12: Full splitting scheme: comparison of the errors $\varepsilon_{p_{2}}$ for different time steps

\section{References}

[1] Alexsson, O., Blaheta, R., Byczanski, P.: Stable discretization of poroelastiicty problems and efficient preconditioners for arising saddle point type matrices. Comput. Vissualizat. in Sci. 15(4), 191-2007 (2013)

[2] Alnæs, M.S., Blechta, J., Hake, J., Johansson, A., Kehlet, B., Logg, A., Richardson, C., Ring, J., Rognes, M.E., Wells, G.N.: The fenics project version 1.5. Archive of Numerical Software 3(100), 9-23 (2015)

[3] Armero, F., Simo, J.C.: A new unconditionally stable fractional step method for non-linear coupled thermomechanical problems. International Journal for $\mathrm{Nu}-$ merical Methods in Engineering 35(4), 737-766 (1992)

[4] Bai, M., Elsworth, D., Roegiers, J.C.: Multiporosity/Multipermeability Approach to the Simulation of Naturally Fractured Reservoirs. Water Resources Research (29), 1621-1633 (1993)

[5] Balay, S., Abhyankar, S., Adams, M.F., Brown, J., Brune, P., Buschelman, K., Dalcin, L., Eijkhout, V., Gropp, W.D., Kaushik, D., Knepley, M.G., McInnes, L.C., Rupp, K., Smith, B.F., Zampini, S., Zhang, H.: PETSc Web page. http: //www.mcs.anl.gov/petsc (2015)

[6] Barenblatt, G.I., Zheltov, I.P., Kochina, I.N.: Basic concepts in the theory of seepage of homogeneous liquids in fissured rocks [strata]. Journal of Applied Mathematics and Mechanics 24(5), 1286-1303 (1960) 
[7] Beskos, D.E., Aifantis, E.C.: On the theory of consolidation with double porosityII. International Journal of Engineering Science 24(11), 1697-1716 (1986)

[8] Biot, M.A.: General theory of three dimensional consolidation. Journal of Applied Physics 12(2), 155-164 (1941)

[9] Boal, N., Gaspar, F.J., Lisbona, F.J., Vabishchevich, P.N.: Finite-difference analysis of fully dynamic problems for saturated porous media. Journal of Computational and Applied Mathematics 236(6), 1090-1102 (2011)

[10] Boal, N., Gaspar, F.J., Lisbona, F.J., Vabishchevich, P.N.: Finite-difference analysis for the linear thermoporoelasticity problem and its numerical resolution by multigrid methods. Mathematical Modelling and Analysis 17(2), 227-244 (2012)

[11] Boal, N., Gaspar, F.J., Lisbona, F.J., Vabishchevich, P.N.: Finite difference analysis of a double-porosity consolidation model. Numerical Methods for Partial Differential Equations 28(1), 138-154 (2012)

[12] Brenner, S.C., Scott, L.R.: The mathematical theory of finite element methods. Springer, New York (2008)

[13] Brezzi, F., Fortin, M.: Mixed and hybrid finite element methods. Springer (1991)

[14] Bukač, M., Yotov, I., Zakerzadeh, R., Zunino, P.: Partitioning strategies for the interaction of a fluid with a poroelastic material based on a nitsches coupling approach. Computer Methods in Applied Mechanics and Engineering 292, 138170 (2015)

[15] Bukač, M., Yotov, I., Zunino, P.: An operator splitting approach for the interaction between a fluid and a multilayered poroelastic structure. Numerical Methods for Partial Differential Equations 31(4), 1054-1100 (2015)

[16] Bychenkov, Y.V., Chizhonkov, E.V.: Iterative methods for solving saddle problems (in Russian). Binom, Moscow (2010)

[17] Gaspar, F.J., Gracia, J.L., Lisbona, F.J., Vabishchevich, P.N.: A stabilized method for a secondary consolidation biot's model. Numerical Methods for Partial Differential Equations 24(1), 60-78 (2008)

[18] Gaspar, F.J., Grigoriev, A.V., Vabishchevich, P.N.: Explicit-implicit splitting schemes for some systems of evolutionary equations. Internat. J. of Num. Analys. Model. 11(2), 346-357 (2014)

[19] Gaspar, F.J., Lisbona, F.J.: An efficient multigrid solver for a reformulated version of the poroelasticity system. Computer Methods in Applied Mechanics and Engineering 196(8), 1447-1457 (2007)

[20] Geuzaine, C., Remacle, J.F.: Gmsh: A 3-d finite element mesh generator with built-in pre-and post-processing facilities. International Journal for Numerical Methods in Engineering 79(11), 1309-1331 (2009) 
[21] Hernandez, V., Roman, J.E., Vidal, V.: SLEPc: A scalable and flexible toolkit for the solution of eigenvalue problems. ACM Trans. Math. Software 31(3), 351-362 (2005)

[22] Jha, B., Juanes, R.: A locally conservative finite element framework for the simulation of coupled flow and reservoir geomechanics. Acta Geotechnica 2(3), 139153 (2007)

[23] Khaled, M.Y., Beskos, D.E., Aifantis, E.C.: On the theory of consolidation with double porosity-III A finite element formulation. International journal for numerical and analytical methods in geomechanics 8(2), 101-123 (1984)

[24] Kim, J., Tchelepi, H., Juanes, R.: Stability and convergence of sequential methods for coupled flow and geomechanics: Drained and undrained splits. Computer Methods in Applied Mechanics and Engineering 200(2324), 2094 - 2116 (2011)

[25] Kim, J., Tchelepi, H., Juanes, R.: Stability and convergence of sequential methods for coupled flow and geomechanics: Fixed-stress and fixed-strain splits. Computer Methods in Applied Mechanics and Engineering 200(1316), 1591 - 1606 (2011)

[26] Knabner, P., Angermann, L.: Numerical methods for elliptic and parabolic partial differential equations. Springer Verlag, New York (2003)

[27] Kolesov, A.E., Vabishchevich, P.N., Vasileva, M.V.: Splitting schemes for poroelasticity and thermoelasticity problems. Computers \& Mathematics with Applications 67(12), 2185-2198 (2014)

[28] Logg, A., Mardal, K.A., Wells, G.N., et al.: Automated Solution of Differential Equations by the Finite Element Method. Springer (2012)

[29] Logg, A., Wells, G.N.: Dolfin: Automated finite element computing. ACM Transactions on Mathematical Software 37(2) (2010)

[30] Marchuk, G.I.: Splitting and alternating direction methods. In: P. Ciarlet, J.L. Lions (eds.) Handbook of Numerical Analysis, pp. 197-462. North-Holland (1990)

[31] Mikelić, A., Wheeler, M.F.: Convergence of iterative coupling for coupled flow and geomechanics. Computational Geosciences 17(3), 455-461 (2012)

[32] Mikhlin, S.G.: The spectrum of a family of operators in the theory of elasticity. Russian Mathematical Surveys 28(3), 45 (1973)

[33] Phillips, P., Wheeler, M.: Overcoming the problem of locking in linear elasticity and poroelasticity: an heuristic approach. Computational Geosciences 13(1), 5$12(2008)$

[34] Samarskii, A.A.: The theory of difference schemes. Marcel Dekker, New York (2001) 
[35] Samarskii, A.A., Matus, P.P., Vabishchevich, P.N.: Difference schemes with operator factors. Kluwer (2002)

[36] Vabishchevich, P.N.: Additive operator-difference schemes. Splitting schemes. de Gruyter (2014)

[37] Vabishchevich, P.N., Vasileva, M.V., Kolesov, A.E.: Splitting scheme for poroelasticity and thermoelasticity problems. Computational Mathematics and Mathematical Physics 54(8), 1305-1315 (2014)

[38] Wheeler, M., Xue, G., Yotov, I.: Coupling multipoint flux mixed finite element methodswith continuous galerkin methods for poroelasticity. Computational Geosciences 18(1), 57-75 (2013)

[39] Wheeler, M.F., Gai, X.: Iteratively coupled mixed and galerkin finite element methods for poro-elasticity. Numerical Methods for Partial Differential Equations 23(4), 785-797 (2007)

[40] Wilson, R.K., Aifantis, E.C.: On the theory of consolidation with double porosity. International Journal of Engineering Science 20(9), 1009-1035 (1982) 